\title{
A New Strain of Potato Carlavirus M
}

T. D. Cavileer, Department of Plant, Soil, and Entomological Sciences, University of Idaho, Moscow, ID 838442339; R. C. Clarke, formerly with Idaho Crop Improvement Association, Idaho Falls, ID 83405-1139; D. L. Corsini, USDA-ARS, Aberdeen, ID 83210; and P. H. Berger, Department of Plant, Soil, and Entomological Sciences, University of Idaho

\begin{abstract}
Cavileer, T. D., Clarke, R. C., Corsini, D. L., and Berger, P. H. 1998. A new strain of potato carlavirus M. Plant Dis. 82:98-102.

In 1994, potato samples for certification from Idaho seed fields reacted in enzyme-linked immunosorbent assay (ELISA) tests to a polyclonal potato carlavirus M (PVM) antiserum. Sample affinity to the antiserum was lower than control samples. Furthermore, ELISA-positive samples were obtained from both symptomatic as well as asymptomatic plants. A complementary DNA library was prepared using both reverse transcription-polymerase chain reaction and primers based on published PVM sequences, or oligo $\mathrm{d}(\mathrm{T})$ primed reverse transcribed sequences. The nucleotide sequence was determined for the $3^{\prime}$-terminus of the genome. Putative coat protein amino acid sequence was compared to published PVM and potato virus S coat protein sequences. While this new isolate is likely a strain of PVM, it is significantly different from known PVM coat protein sequences in the amino terminus region. These differences may explain the poor reactivity to other PVM antisera and suggest that it is a new strain of PVM, which we have designated PVM-ID.
\end{abstract}

Detection and elimination of viruses in potato seed stock is paramount to potato health and production worldwide. Consequently, seed certification programs are important in detecting, quantifying, and eliminating major potato viruses. When new virus isolates or strains occur, the spectrum of standard tests must be updated to provide the most efficacious detection possible.

Late in 1994, plants of potato breeding selection A82705-1R sent to New Brunswick, Canada, tested positive by enzymelinked immunosorbent assay (ELISA) for potato carlavirus $\mathrm{M}$ (PVM). No symptoms of PVM infection were observed among previous lots grown in Idaho. However, during subsequent grow-out tests in California, two nuclear seed-lots produced from pre-nuclear stocks of this variety in southern Idaho contained plants with mosaic symptoms. PVM-infected plants were confirmed after testing with antisera for various mosaic-causing viruses.

These reports led us to investigate this isolate of PVM, characterize the virus, and produce a reliable antiserum to augment the potato seed certification tests in Idaho.

Corresponding author: P. H. Berger

E-mail: pberger@uidaho.edu

PVM-ID sequence has been deposited with GenBank, Accession \# AF023877.

Accepted for publication 1 October 1997.

Publication no. D-1997-1120-01R

(C) 1998 The American Phytopathological Society

\section{MATERIALS AND METHODS}

Virus source, isolation and purification. Virus particles were initially extracted from infected potato leaf samples of A82705-1R. Because field samples contained mixed infection of potato virus $S$ (PVS) and PVM, subsequent virus extractions were made from plants grown in sue culture samples were generated and provided by L. Ewing, Idaho Nuclear Seed Potato Program.

Plant tissue infected with PVM was titurated in three volumes of extraction buffer (100 mM Na-borate, pH 8.2, $100 \mathrm{mM}$ EDTA, 2\% [wt/vol] polyvinylpyrrolidone [PVP-40], 0.1\% $\beta$-mercaptoethanol), expressed through cheesecloth, and centrifuged $10 \mathrm{~min}$ at $16,270 \times g$. The resulting with $\mathrm{NaCl}$ and $6 \%(\mathrm{wt} / \mathrm{vol})$ polyethylene glycol (MW 8000) and stirred for $2 \mathrm{~h}$ at room temperature. The precipitate was collected by centrifugation for $20 \mathrm{~min}$ at $16,270 \times g$ at $4^{\circ} \mathrm{C}$. The pellet was resuspended overnight in $20 \mathrm{ml}$ of $10 \mathrm{mM} \mathrm{Na}$ phosphate buffer, $\mathrm{pH} 7.5$. trifugation for $10 \mathrm{~min}$ at $16,270 \times g$ at $4^{\circ} \mathrm{C}$, then overlaid on a $20 \%$ sucrose cushion in $100 \mathrm{mM}$ Na-phosphate buffer, pH 7.5. High speed centrifugation was performed for $1 \mathrm{~h}$ at $240,000 \times g$ at $4^{\circ} \mathrm{C}$ using a Type 70Ti rotor (Beckman Instruments, Inc., Palo Alto, CA). The resulting pellet was resuspended in $10 \mathrm{mM}$ Na-phosphate buffer, clarified by low speed centrifugation, and overlaid on a $30 \%$ sucrose cushion. After the second high speed centrifutissue culture that tested free of PVS. Tissupernatant was brought up to $200 \mathrm{mM}$

The resuspension was clarified by cen- gation, the resuspended pellet was clarified by low speed centrifugation and subjected to cesium chloride density gradient centrifugation (density $\approx 1.28 \mathrm{~g} / \mathrm{ml}$ ). A single migrating band was collected after centrifugation for $8 \mathrm{~h}$ at $240,000 \times g$ at $20^{\circ} \mathrm{C}$ using a Sorvall TV-865 rotor (Du Pont Company, Wilmington, DE). The collected band was diluted in $10 \mathrm{mM}$ Na-phosphate buffer, $\mathrm{pH} 7.5$, pelleted by high speed centrifugation $\left(1 \mathrm{~h}\right.$ at $240,000 \times g$ at $\left.4^{\circ} \mathrm{C}\right)$, and resuspended in 0.1 to $0.5 \mathrm{ml}$ of $10 \mathrm{mM} \mathrm{Na}$ phosphate buffer, $\mathrm{pH} 7.5$, containing $0.04 \%$ sodium azide.

RNA extraction. Genomic RNA was extracted from virions by combining 500 $\mu \mathrm{g} / \mathrm{ml}$ of purified virus with $5 \mu \mathrm{l} 20 \%$ sodium dodecyl sulfate (SDS) and $5 \mu 1$ of proteinase $\mathrm{K}(5 \mathrm{mg} / \mathrm{ml})$ in a microcentrifuge tube and incubating the mixture at $37^{\circ} \mathrm{C}$ for $15 \mathrm{~min}$. An additional $25 \mu \mathrm{l}$ of $20 \%$ SDS was added and the mixture heated to $65^{\circ} \mathrm{C}$ for $5 \mathrm{~min}$. The mixture was extracted with an equal volume of TrisEDTA (TE) $8(10 \mathrm{mM}$ Tris, $1 \mathrm{mM}$ EDTA, $\mathrm{pH}$ 8.0)-saturated phenol at $65^{\circ} \mathrm{C}$, vortexed briefly, and centrifuged in a benchtop centrifuge for $5 \mathrm{~min}$ at room temperature. This step was repeated three times. The upper phase was transferred to a clean tube and extracted five times with chloroform (24:1, chloroform:isoamyl alcohol), centrifuged as before, and the upper phase transferred to a clean tube. The genomic RNA was precipitated with $125 \mu \mathrm{l}$ of $7.5 \mathrm{M}$ ammonium acetate, $\mathrm{pH} 5.2$, and $750 \mu \mathrm{l} 100 \%$ ethanol, and incubated for $2 \mathrm{~h}$ at $-80^{\circ} \mathrm{C}$. The precipitated RNA was collected by centrifuging the solution for $30 \mathrm{~min}$ at $16,270 \times g$. The resulting pellet was washed once with $70 \%$ ethanol, centrifuged for $15 \mathrm{~min}$ at $16,270 \times g$, and resuspended in $50 \mu \mathrm{l}$ diethyl pyrocarbonate (DEPC)-treated water.

cDNA synthesis. Two methods were employed to generate cDNA for use in subsequent procedures. The first involved production of first-strand cDNA from intact virions as previously described (11), and the second from isolated RNA. When doublestranded cDNA was required, reactions from first-strand synthesis using Superscript II (GIBCO BRL, Gaithersburg, MD) were carried through to the second strand cDNA synthesis reactions, as described by the manufacturer's protocol. The resulting blunt-ended, double-stranded cDNA was used in subsequent ligation reactions. 
RT-PCR. Reverse transcription-polymerase chain reaction (RT-PCR) was performed using first-strand cDNA generated from genomic RNA or intact virions as described above. Generally, $4 \mu \mathrm{l}$ of firststrand cDNA was combined on ice with $73.2 \mu 1$ water, $10.0 \mu \mathrm{l} 10 \times$ PCR buffer (GIBCO BRL), $2.5 \mu \mathrm{l} 20 \mu \mathrm{M}$ homologousstrand primer (5'-GGCGATGGGAGATTCAACGAAGAAAG-3'), $2.5 \mu 120 \mu \mathrm{M}$ complementary-strand primer (5'-GGAGTAAAGCCACCTTGGTTACGTC-3'), 6.0 $\mu 125 \mathrm{mM} \mathrm{MgCl}_{2}, 0.8 \mu \mathrm{l} 25 \mathrm{mM}$ dNTP mix, and 5U Taq DNA polymerase (GIBCO BRL). Reactions were overlaid with Chill-out (MJ Research, Watertown, MA) and incubated for $5 \mathrm{~min}$ at $95^{\circ} \mathrm{C}, 5$ $\min$ at $85^{\circ} \mathrm{C}$, and $2 \mathrm{~min}$ at $60^{\circ} \mathrm{C}$. Thirtyfive cycles of $1 \mathrm{~min}$ at $94^{\circ} \mathrm{C}, 1 \mathrm{~min}$ at $60^{\circ} \mathrm{C}$, and $1 \mathrm{~min}$ at $72^{\circ} \mathrm{C}$ were performed, followed by $10 \mathrm{~min}$ at $72^{\circ} \mathrm{C}$. Reactions were held at $9^{\circ} \mathrm{C}$ until assayed.

Cloning. The PCR-amplified fragments were electrophoresed on $1 \%$ agarose, excised, and purified using USB Bioclean MP kit (USB, Cleveland, OH). Purified PCR fragments were cloned into pCRII (Invitrogen Corp., San Diego, CA) and transformed into INV $\alpha \mathrm{F}^{1}$-competent cells. Additionally, oligo d(T) primed cDNA was blunt-end cloned into the EcoRV site of pBSIISK+, and DH10B-competent cells were transformed. Colonies positive for inserts were selected by blue/white screening on agar media supplemented with 100 $\mu \mathrm{g} / \mathrm{ml}$ ampicillin, $40 \mu \mathrm{M}$ isopropyl $\beta$-Dthiogalactopyranoside (IPTG), and $40 \mu \mathrm{m} 5$ bromo-4-chloro-3-indolyl- $\beta$-D-galactopyranoside (Xgal; 9). Subclones were produced by restriction fragment digests and by exonuclease III digestion (9).

Nucleic acid sequencing and multiple sequence alignments. Sequencing reactions were performed using the SequiTherm Cycle Sequencing LongRead Kit (Epicentre Technologies Corporation, Madison, WI) and run on a LI-COR model 4000L Automated DNA Sequencer (LICOR, Inc., Lincoln, NB). Sequence was collected and analyzed using BaseImager 2.20 software (LI-COR, Inc.). Fragment assembly, multiple sequence analysis, and antigenic site predictions were accomplished using individual programs from the Wisconsin GCG 8.0 software package (Genetics Computer Group, Madison, WI).

Predicted amino acid (a.a.) sequences used for comparison were taken from the literature and designated as follows: PVMG (3), PVM-R (7), PVM-Z (12), PVS-An (5), and PVS-O (2). Amino acid sequence alignments were preformed using Clustal V (EMBL Heidelberg, Germany) and displayed using SeqVu 1.0 (Garvan Institute, Sydney, Australia).

Antibody production. Polyclonal antibodies were produced using purified virions as antigen emulsified 1:1 with TitreMax Adjuvant (Vaxcel, Inc., Norcoss, GA), and injecting female New Zealand white rabbits with $100 \mu \mathrm{l}$ in two sites (one subcutaneous and one intramuscular). Serum was collected at one month post-injection, cross-absorbed with healthy potato extract, conjugated to alkaline phosphatase, and stored at $4^{\circ} \mathrm{C}$ with $0.04 \%$ sodium azide.

SDS-PAGE electrophoresis and Western blotting. Samples were prepared by grinding leaf disks ( 1-cm diameter) 1:35 (wt/vol) in disassociation buffer $(2.5 \%$ SDS, 2.5\% $\beta$-mercaptoethonal, 20\% sucrose), boiling for $10 \mathrm{~min}$, and centrifuging in a benchtop microcentrifuge for $4 \mathrm{~min}$. Total plant protein samples were separated on duplicate $12.5 \%$ SDS-polyacrylamide gel electrophoresis (PAGE) gels and elec- trophoresed at $6 \mathrm{~W}$ constant power for $4 \mathrm{~h}$ in a Hoefer Model SE400 Vertical Slab Gel Unit (Hoefer Scientific, San Francisco, CA). One gel was used for staining with Coomassie brilliant blue R-250, and one gel soaked in transfer buffer $(25 \mathrm{mM}$ Tris, $250 \mathrm{mM}$ glycine, $0.05 \% \mathrm{SDS}$ ) for $10 \mathrm{~min}$ and electroblotted to nitrocellulose for $1 \mathrm{~h}$ at 5 watts, using a semi-dry electroblotter (ISS, Natick, MA). Blots were incubated with phosphate buffered saline (PBS) containing 5\% Carnation non-fat milk for $1 \mathrm{~h}$ at room temperature. Primary polyclonal antibody was added at 1:2000 and incubated overnight at room temperature on an orbital shaker. Nitrocellulose blots were

Table 1. Indirect enzyme-linked immunosorbent assay (ELISA) data from potato leaf samples for the Idaho strain of potato carlavirus M (PVM-ID) ${ }^{\mathrm{a}}$

\begin{tabular}{lccccc}
\hline & & \multicolumn{4}{c}{ Antiserum $^{\mathbf{b}}$} \\
\cline { 4 - 6 } Sample & Number & $\mathbf{\# 1}$ & $\mathbf{\# 2}$ & $\mathbf{\# 3}$ & $\boldsymbol{\# 4}$ \\
\hline Collection PVM $^{\mathrm{c}}$ & $\mathrm{n}=3$ & $0.65 \pm 0.07$ & $>2.00$ & $1.76 \pm 0.28$ & $0.06 \pm 0.01$ \\
A82705-1R (virus free) & $\mathrm{n}=6$ & $0.03 \pm 0.01$ & $0.03 \pm 0.02$ & $0.01 \pm 0.00$ & $0.04 \pm 0.03$ \\
A82705-1R (PVS) & $\mathrm{n}=6$ & $0.07 \pm 0.02$ & $0.26 \pm 0.03$ & $0.04 \pm 0.02$ & $0.01 \pm 0.00$ \\
$\begin{array}{l}\text { A82705-1R (PVM-ID) } \\
\text { A82705-1R }\end{array}$ & $\mathrm{n}=6$ & $0.68 \pm 0.18$ & $0.41 \pm 0.16$ & $0.03 \pm 0.02$ & $0.51 \pm 0.03$ \\
(PVM-ID + PVS) & $\mathrm{n}=6$ & $0.72 \pm 0.20$ & $0.51 \pm 0.16$ & $0.05 \pm 0.02$ & $0.42 \pm 0.01$ \\
\hline
\end{tabular}

${ }^{a}$ ELISA data for antiserum \#4 were collected in a separate experiment but are included in the table for comparison with earlier ELISA results. ELISA readings in all experiments were at $405 \mathrm{~nm}$ using alkaline phosphatase. Numbers to right of each value are standard deviations.

b Antiserum \#1, Bioreba Corp. (lot \#110221) South Bend, IN; antiserum \#2, Idaho Crop Improvement Association (Thomas), Idaho Falls, ID; antiserum \#3, Agdia Corp. (lot \#0188) Elkhart, IN; and antiserum \#4, University of Idaho, PSES Dept., Moscow, ID.

${ }^{c}$ Supplied by R. Goth, USDA-ARS, Beltsville, MD; originally collected by E. S. Shultz.

${ }^{\mathrm{d}} \mathrm{PVS}=$ potato virus $\mathrm{S}$.

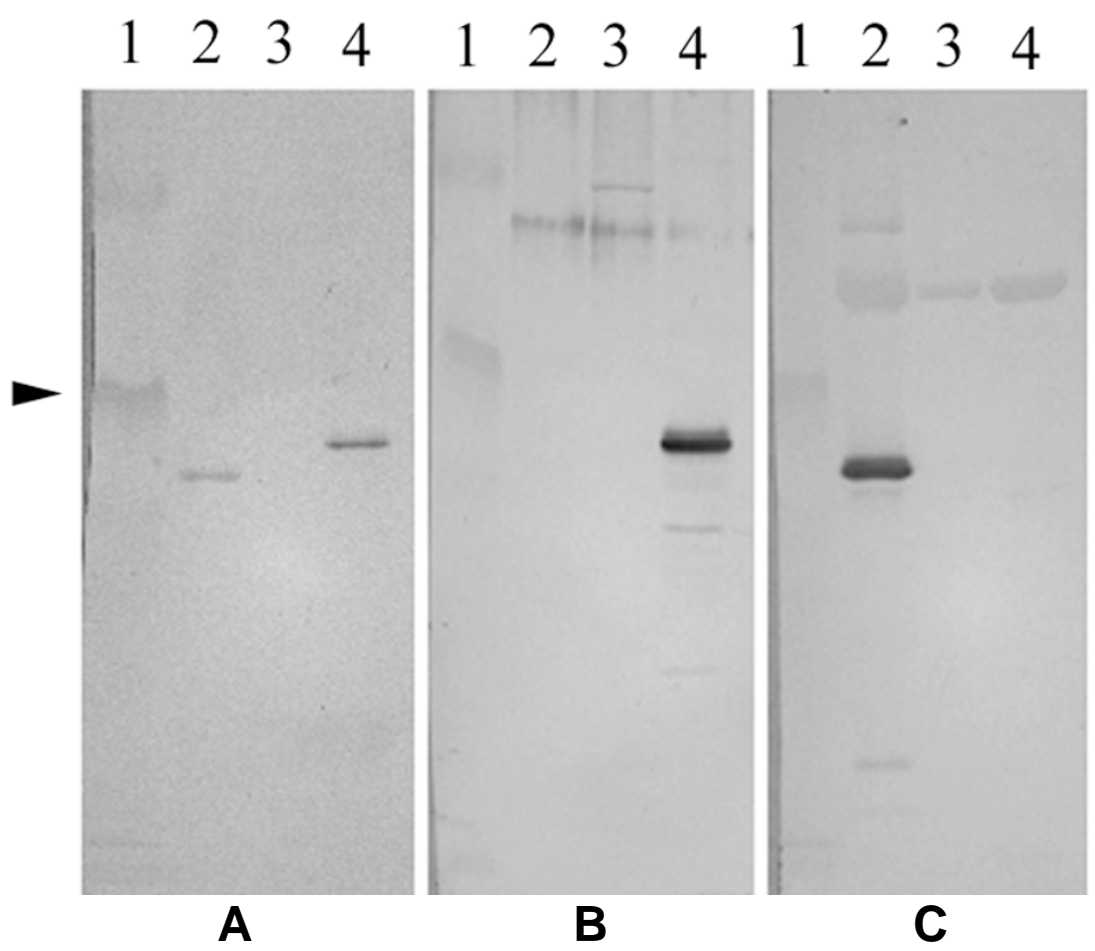

Fig. 1. Replicate Western blots of total protein samples extracted from plant tissue. (A) Blot probed with potato carlavirus M (PVM) antiserum (Idaho Crop Improvement Association [ICIA]); (B) blot probed with PVM-ID antiserum, and (C) blot probed with potato virus S (PVS) antiserum (ICIA). Arrow indicates $41.8-\mathrm{kDa}$ prestained marker. Lanes in all three gels are (1) kaleidoscope markers (BioRad, Richmond, CA), (2) PVS-infected tobacco, (3) non-infected potato, and (4) PVM-ID infected potato. 
washed twice in PBS and once in Trisbuffered saline (TBS) for $10 \mathrm{~min}$ at room temperature. Blots were then incubated for $5 \mathrm{~h}$ in TBS (containing 5\% Carnation nonfat milk) and goat anti-rabbit alkaline phosphatase (1:2000) or goat anti-sheep alkaline phosphatase (1:2000) for PVM and PVS, respectively. The blot was washed twice with TBS and once with AP buffer $(100 \mathrm{mM} \mathrm{NaCl}, 50 \mathrm{mM} \mathrm{MgCl} 2,100$ $\mathrm{mM}$ Tris, $\mathrm{pH}$ 9.5) for $10 \mathrm{~min}$ at room temperature. Color development was accomplished using Western Blue stabilized substrate (Promega, Corp., Madison, WI).

\section{RESULTS}

Virus extractions from field samples contained both PVM and PVS. After passage in tissue culture, using techniques to produce potato tissue free of PVS, plants were obtained with only PVM, and this isolate was designated PVM-ID.

Early serological tests by the Idaho Crop Improvement Association (ICIA) did not detect PVM in pre-nuclear mini-tubers produced and released by the Nuclear Seed Program. PVM symptoms were not observed in greenhouse grow-out tests established for the potato certification program.
After receiving reports from Canada of PVM infection in potato line A82705-1R, symptomatic plants from the California field grow-out plots were tested by ELISA and found positive for PVM infection. Reactions were not strong, ranging from optical density (OD) 405 of 0.2 to 0.5 (data not shown), with positive controls containing the type culture of PVM reading 2.0.

Additional ELISA tests were run on symptomatic tissue using antisera from Agdia (Elkhart, IN), ICIA (Idaho Falls, ID), and Bioreba Inc. (South Bend, IN).

$1 / 1$

$31 / 11$

ATG GGA GAT TCA ACA AAG AAA ATT GAA GTT GCC AAA GAA GCT GGA ACA TCG CAA GCG GCC $\begin{array}{lllllllllllllllllllll}M & G & D & S & T & K & K & I & E & V & A & K & E & A & G & T & S & Q & A & A\end{array}$ $61 / 21 \quad 91 / 31$

AAG GGG AAC AGA CCA CTA CCT ACG GCG GCA GAA TTT GAA GGT GAT GAC ACC TCT GGA GAT $\begin{array}{lllllllllllllllllllll}K & G & N & R & P & L & P & T & A & A & E & F & E & G & D & D & T & S & G & D\end{array}$ $121 / 41$

GCT AGT GTC CGC AAT GCT GAG GCA AAT GAG GAA GCG TCA CTG GAG CGC AGA TTG AAT AGC $\begin{array}{lllllllllllllllllllll}A & S & V & R & N & A & E & A & N & E & E & A & S & L & E & R & R & L & N & S\end{array}$ $181 / 61 \quad 211 / 71$

TTG CGT GAG TTT CTA CGT GAG CGA AGA GGC GCC ATA CGG GTA ACC AAC CCT GGT TTG GAG $\begin{array}{llllllllllllllllllllll}\mathrm{L} & \mathrm{R} & \mathrm{E} & \mathrm{F} & \mathrm{L} & \mathrm{R} & \mathrm{E} & \mathrm{R} & \mathrm{R} & \mathrm{G} & \mathrm{A} & \mathrm{I} & \mathrm{R} & \mathrm{V} & \mathrm{T} & \mathrm{N} & \mathrm{P} & \mathrm{G} & \mathrm{L} & \mathrm{E}\end{array}$ $241 / 81 \quad 271 / 91$

ACT GGC CGT CCA CGA CTC AAG CTT GCA GAC GAC ATG CGC CCG GAT CCA ACA AAT CCT TAC $\begin{array}{lllllllllllllllllllll}T & \mathrm{G} & \mathrm{R} & \mathrm{P} & \mathrm{R} & \mathrm{L} & \mathrm{K} & \mathrm{L} & \mathrm{A} & \mathrm{D} & \mathrm{D} & \mathrm{M} & \mathrm{R} & \mathrm{P} & \mathrm{D} & \mathrm{P} & \mathrm{T} & \mathrm{N} & \mathrm{P} & \mathrm{Y}\end{array}$ $301 / 101 \quad 331 / 111$

AAC AGG CCG TCG CTT GAG GCA CTG AGC AGA ATT AAA CCT ATA GCT GTG TCA AAC AAT ATG $\begin{array}{lllllllllllllllllllll}\mathrm{N} & \mathrm{R} & \mathrm{P} & \mathrm{S} & \mathrm{L} & \mathrm{E} & \mathrm{A} & \mathrm{L} & \mathrm{S} & \mathrm{R} & \mathrm{I} & \mathrm{K} & \mathrm{P} & \mathrm{I} & \mathrm{A} & \mathrm{V} & \mathrm{S} & \mathrm{N} & \mathrm{N} & \mathrm{M}\end{array}$ $361 / 121$ $391 / 131$

GCC ACA TCT GAG GAC ATG ATG CGC ATC TAT GTC AAT CTT GAA GGT TTG GgG GTG CCA ACT $\begin{array}{lllllllllllllllllllll}A & T & S & E & D & M & M & R & I & Y & V & N & L & E & G & L & G & V & P & T\end{array}$ $421 / 141$ GAG TAC GTG CAG CAA GTG GTG ATC CAA GCG GTG CTT TTC TGC AAA GAT GCA AGT AGC TCG $\begin{array}{llllllllllllllllllllll}E & \mathrm{Y} & \mathrm{V} & \mathrm{Q} & \mathrm{Q} & \mathrm{V} & \mathrm{V} & \mathrm{I} & \mathrm{Q} & \mathrm{A} & \mathrm{V} & \mathrm{L} & \mathrm{F} & \mathrm{C} & \mathrm{K} & \mathrm{D} & \mathrm{A} & \mathrm{S} & \mathrm{S} & \mathrm{S}\end{array}$ $481 / 161 \quad 511 / 171$

GTG TAT CTC GAT CCA CGC GGC TCC TTC GAA TGG CCA AGG GGT GCA ATC ACT GCA GAT GCG $\begin{array}{lllllllllllllllllllll}\mathrm{V} & \mathrm{Y} & \mathrm{L} & \mathrm{D} & \mathrm{P} & \mathrm{R} & \mathrm{G} & \mathrm{S} & \mathrm{F} & \mathrm{E} & \mathrm{W} & \mathrm{P} & \mathrm{R} & \mathrm{G} & \mathrm{A} & \mathrm{I} & \mathrm{T} & \mathrm{A} & \mathrm{D} & \mathrm{A}\end{array}$ $541 / 181 \quad 571 / 191$

GTG CTG GCT GTG ATG AAG AAA GAC GCA GAG ACT CTG CGC AGA GTC TGT AGg CTG TAC GCG $\begin{array}{lllllllllllllllllllll}\mathrm{V} & \mathrm{L} & \mathrm{A} & \mathrm{V} & \mathrm{M} & \mathrm{K} & \mathrm{K} & \mathrm{D} & \mathrm{A} & \mathrm{E} & \mathrm{T} & \mathrm{L} & \mathrm{R} & \mathrm{R} & \mathrm{V} & \mathrm{C} & \mathrm{R} & \mathrm{L} & \mathrm{Y} & \mathrm{A}\end{array}$ $601 / 201$ 631/211

CCC GTC ACA TGG AAT CAC ATG CTG GCG CAC AAT TCC CCA CCA GCG GAC TGG GCG GCT ATG $\begin{array}{lllllllllllllllllllll}\mathrm{P} & \mathrm{V} & \cdot \mathrm{T} & \mathrm{W} & \mathrm{N} & \mathrm{H} & \mathrm{M} & \mathrm{L} & \mathrm{A} & \mathrm{H} & \mathrm{N} & \mathrm{S} & \mathrm{P} & \mathrm{P} & \mathrm{A} & \mathrm{D} & \mathrm{W} & \mathrm{A} & \mathrm{A} & \mathrm{M}\end{array}$ $661 / 221 \quad 691 / 231$

GGT TTC CAA TAT GAA GAT AGA TTC GCA GCC TTT GAC TGC TTT GAC TAC GTG GAG AAC ACA $\begin{array}{lllllllllllllllllllll}G & F & Q & Y & E & D & R & F & A & A & F & D & C & F & D & Y & V & E & N & T\end{array}$ $721 / 241 \quad 751 / 251$

GCT GCA GTT CAA CCA CTG GAA GGG CTG ATA CGC CGT CCT ACC CCA AGA GAG AAG GTT GCG $\begin{array}{lllllllllllllllllllll}A & A & V & Q & P & L & E & G & L & I & R & R & P & T & P & R & E & K & V & A\end{array}$ $781 / 261 \quad 811 / 271$ CAT AAC ACT CAC AAG GAC ATA GCT CTG AGG GGG GCC AAT CGC AAT CAG GTG TTT GGT TCG $\begin{array}{llllllllllllllllllll}\mathrm{H} & \mathrm{N} & \mathrm{T} & \mathrm{H} & \mathrm{K} & \mathrm{D} & \mathrm{I} & \mathrm{A} & \mathrm{L} & \mathrm{R} & \mathrm{G} & \mathrm{A} & \mathrm{N} & \mathrm{R} & \mathrm{N} & \mathrm{Q} & \mathrm{V} & \mathrm{F} & \mathrm{G} & \mathrm{S}\end{array}$ $841 / 281 \quad 871 / 291$ CTC AAT GCT GAG GTA ACT GGA GGT ATG AAT GGT CCC GAG CTC ACT AGG GAT TTC GGC AAG $\begin{array}{lllllllllllllllllllll}\mathrm{L} & \mathrm{N} & \mathrm{A} & \mathrm{E} & \mathrm{V} & \mathrm{T} & \mathrm{G} & \mathrm{G} & \mathrm{M} & \mathrm{N} & \mathrm{G} & \mathrm{P} & \mathrm{E} & \mathrm{L} & \mathrm{T} & \mathrm{R} & \mathrm{D} & \mathrm{F} & \mathrm{G} & \mathrm{K}\end{array}$ $901 / 301$ TCG AAT AAC AAA TGA

$S \quad \mathrm{~N} N$ *

Fig. 2. Nucleic acid sequence and predicted amino acid sequence of potato carlavirus M-ID coat protein region. 
The results for PVM-ID varied widely. The Agdia antiserum did not react. The ICIA antiserum reacted but provided low readings. The Bioreba antiserum gave a more clear-cut reaction than was observed with the ICIA antiserum (Table 1). PVM-ID polyclonal antibody, cross-absorbed with healthy potato extract and conjugated to alkaline phosphatase, detected PVM-ID and did not cross-react with the type strain of PVM or PVS in subsequent ELISA tests (Table 1).

Western analysis of total protein samples paralleled ELISA data and showed that the source of antibody was critical for detec-
PVM-ID

PVM-G

PVM-R

PVM-Z

PVS-An

PVS-O

PVM-ID

PVM-G

PVM-R

PVM-Z

PVS-An

PVS-O

PVM-ID

PVM-G

PVM-R

PVM-Z

PVS-An

PVS-O

PVM-ID

PVM-G

PVM-R

PVM-Z

PVS-An

PVS-O

PVM-ID

PVM-G

PVM-R

PVM-Z

PVS-An

PVS-O

PVM-ID

PVM-G

PVM-R

PVM-Z

PVS-An

PVS-O

PVM-ID

PVM-G

PVM-R

PVM-Z

PVS-An

PVS-O

PVM-ID

PVM-G

PVM-R

PVM-Z

PVS-An

PVS-O

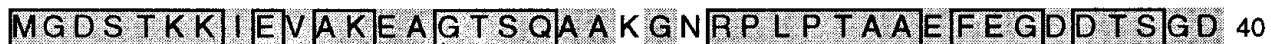
MGDS TKKA E T A KDV GT S QEKREARP LP TA A DEFEGKDT SED 40 MGDSTKKA E TAKDEGTS QERREARP LP TAADEE GKDT S EN 40 MGDS TKKAEETA K D E G T S QER RREA RPLP T A A D FE G]KDT S E N 40 M- - PPIK PDPSS S G A PIOAMQPAPPPRA - E G- - - HMY 30 M- - P P KJPDPTS S GETPQQA I P LA]PBPRNVEE - . - - HR I 31
\end{abstract}

A S VRNAIEANEEASLERRLN SLREFLRERRGA IRV TNPGLE 80 41 TD GRA A DAD GEMSLERR LDSLREFLRERRGA IRV TNP GLE 80 41 TD GRAA D A D GEMS LERR LDSLRE FLRERRGA IRVTNPGLE 80 41 T D GRAA A D A D GEMS LERR L D S LRE FLRERR G A IRV TNP G LE 80 31 A QPEGPGQNEEAMLERAL I RLTELMA TKRIHNS TLSN ISFE 70 32 GPNQGHGQNEEA MLE]QR L I RLL IELMASKEHNS TLS I A FE 71

T GRPRL K [ADDDMRPDP TNPYNRPS]LEALSR IKP IA] SNNM 120 T GRPRL Q LA EN MRP DP T NP YNRPS IEALSRI KP IA 1 SNNM 120 T GRPRLQLA ENMRPDP TNP YNRPS I EA L SR I KP I A I SNNM 120 T GRPR L QLLAENMRPDP TNP YNRPS I EA LSR I KP IA] I SNNM 120 TGRPSLE P TPEMRRNP EN P Y SRTFS I DEL FKME IRS V S NNM 110

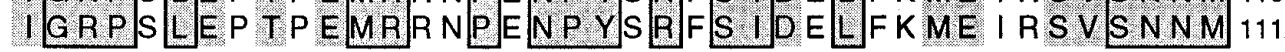

121

121

121

121

111

112

161

161

161

161

151

152

201

201

201

201

190

191

241

241

241

241

230

231

278

278

278

278

267

271

A TSEDMMR I YVNLEGL GVP TEYVQQVVI QAVLFCKDA SS S 160 A T SEDMMR I YVNLEGLGVP TEHVQQVV I QAVLFCKDASSS 160 A T SEDMMR I YVNLEGL GVP TEHVQQVVIQAVL FCKDASSS 160 A T S EDMMR I Y V N LE GL GVP TEHVQQVVI QAV L FCKDA S S 160 A NTE QMA Q I T A D IA G L G VP T E HV A GVI LKVVIIMCA SVS S S 150 A N TE QMA Q I T A D I A GL G V P T E HV A GVII L KVVVI MCA SV D S S 151

VI L DPRGS FEWPRGA I T A D A V LA VMKK DAE TLRR V CR LYA 200 V F L DPR GS FEWPRGA I T A D A V LA VLKK D A E T L R R V C R L Y A 200 V F L D P R GS FE WPRGA I T A D V L A VLKK D A E T LR V C L Y A 200 VFLDPR GS FEWPRGA I T A D A V LA VLKK D A E T LR V CR L Y A 200

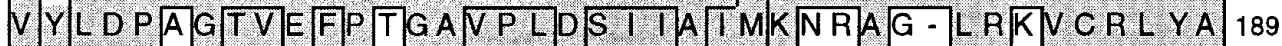
V Y L D P A G TVE F P T G A V P L DSS I I A I MKN RA G - LR KV C A L Y A 190

PV TWNHMLA HNS PPADWA A MGFQYEDRFA A FDCFDYVENT 240 PV TWNHML TH NAP P A EWA A MG F Y Y D D FAPFD C F D Y V E N T 240 PV TWN HML TH NAP P A DWA A M F Q Y E D R F A A F D F F YV E N T 240 PV TWNHMLTT NAP P A DWA A M F Q YEDRFA A F D F D YVENT 240 P V VWNTM LV QN R P P STDWQA M G F OWNAR F A A FDTIF D Y V TTNG 229

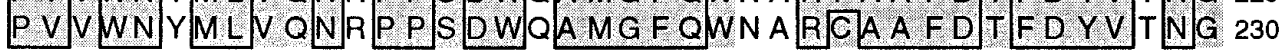

A AVQPLEGL IRRP TPREKVAHN TH- - KDIALLRANRN QV 277 A A V QP L E G L I R R P T REKVA H N TH- - KD I AVR GA NR N QV 277 A A V QP LE G L I RRP TPREKV A H N TH. - KD I AVRGANR NQV 277 A A VQP LE G I RRP TPREKVAHNTH- - KD I AVRGANR NQV 277 A A TOQPVE G L I R R P T P E]E[T TA H NATH - - KS MA I DKS]N R NER 266 A A V QP VE G L IR R P T P E E T I A H NA HN A HKS MA I D K S NA NE R 270

FGS LNAEVT G GMNGPE L TRDFGKSNNK FSS LNAEVTGGMNGPE L TRD YVKSNRK FSS L NAEVTGGMNGPE L TRD YVK SNRK FIS L NAEVT G GMNGPE L TRDYVK SNRK 304 LANTNVEMTGGMLGAE IVRRHRNA INQ LA NTNVE Y T G GMLLAE I V BNHRNA I NQ

Fig. 3. Alignment of coat protein sequence of potato carlavirus M (PVM)-ID with three sequences of PVM and two strains of potato virus S. Homologous amino acids are identified by shading; boxed amino acids indicate identity for each position in the amino acid chain. 
tion of this virus. When protein samples were probed with antibodies raised against the type strain of PVM, detection was sporadic, and cross-reaction with proteins from PVS often was observed (Fig. 1A and $1 \mathrm{C}$, respectively). Antibodies raised to purified PVM-ID (Fig 1B) detected protein of the expected size $(\sim 34 \mathrm{kDa})$ and only faintly cross-reacted with PVS.

Initial cloning efforts from cDNA from intact virions using custom primers yielded a fragment of 804 base pairs (bp). Sequence data confirmed that the fragment corresponded to the $5^{\prime}$-terminus and core of the coat protein region of PVM, but lacked the 3'-terminus expected from published sequences. Subsequent clones generated from oligo $d(T)$-primed cDNA revealed the entire coat protein sequence (Fig. 2) of 928 bp coding for 304 amino acids, the sequence encompassing the triple block genes, and the $3^{\prime}$ open reading frame normally associated with carlaviruses (data not shown). Sequence alignment of the putative coat protein translation product (33.6 kDa) show that PVM-ID is more closely related to other PVM sequences (92\% similarity, $88 \%$ identity) than to PVS sequences $(57-58 \%$ similarity, $46 \%$ identity; Fig. 3). Within the first 50 a.a. of the coat protein region, similarity to other PVM sequences drops to 70 to $72 \%$.

\section{DISCUSSION}

PVM-ID is typical of many latent potato viruses, in that symptoms range from very slight to severe depending on the potato variety and environmental conditions. Symptom expression of PVM-ID was not noticeably different from that of ordinary PVM. Typical symptoms included mottling, crinkling, and leafrolling, and stunting of shoots.

Antisera from Agdia, ICIA, and Bioreba varied widely in ELISA and Western blot tests of PVM-ID in potatoes. These tests suggested that there was a new strain of PVM in the released material. It also became clear that the problem of detection of PVM-ID was rooted in the latency of the virus and in the source of antisera, with varying levels of detection correlating with the source of antisera. Because of this variability in reaction to antisera raised against type PVM antigen, we produced antiserum to PVM-ID. This new antiserum can reliably detect PVM-ID and distinguish it from the type strain of PVM.

Sequence data revealed a greater homology between published PVM sequences and PVM-ID than with PVS, clearly indicating that it is an isolate of PVM. Genomic structure is typical of carlavirus genome organization, with evidence of the replicase motif in the RNA-dependent RNA polymerase (RdRp) gene, an overlapping triple block gene set followed by the coat protein gene, and ending with a $3^{\prime}-$ end open reading frame. Protein alignments of published carlavirus coat protein sequences place most of the heterogeneity within the amino terminus (data not shown). This region of heterogeneity falls within the range normally associated with plant virus coat protein antigenic sites $(4,8)$, and may explain why antisera to the ordinary strain of PVM failed or only marginally detected PVM-ID using ELISA.

Virus speciation and strain delineation is difficult given the polythetic nature of viruses. However, while there is no criterion concerning classification of virus species and strains, it is widely accepted that delineation based on a combination of molecular, serological, and biological properties can be both useful and convenient. Properties such as the degree of coat protein sequence identity (approximately 90 to $99 \%$ for strains) and serological relationships have been used in the past to establish membership in virus species groups and to distinguish strains $(1,6,10)$. Discounting the normally variable and latent nature of PVM symptom expression in general, we believe it is appropriate to use the serological and molecular properties of this isolate to designate PVM-ID as a new strain of PVM.

We have presented evidence that potato breeding selection A82705-1R contained PVM, that there is a difference in coat protein sequence from published PVM isolates, especially within the first 50 a.a. normally associated with major antigenic sites, and that there are significant differences among various PVM antisera. We believe these factors indicate that PVM-ID is a distinct strain.

The origin of this new strain has not been established, but subsequent testing of breeding germplasm detected the virus in two introduced potato lines. They probably became the initial source of inoculum, which spread to the breeding selection A82705-1R. The fact that this strain is normally latent under summer growing conditions made visual identification unlikely.

\section{LITERATURE CITED}

1. Barnett, O. W. 1992. A summary of potyvirus taxonomy and definitions. Arch. Virol. Suppl. 5:435-444.

2. Foster, G. D., and Mills, P. R. 1992. The 3'nucleotide sequence of an ordinary strain of potato virus S. Virus Genes 6:213-220.

3. Gramstat, A., Courtpozanis, A., and Rohde, W. 1990. The 12-kDa protein of potato virus M displays properties of a nucleic acid-binding regulatory protein. FEBS Lett. 276:34-38.

4. Jordan, R. 1992. Potyviruses, monoclonal antibodies, and antigenic sites. Arch. Virol. Suppl. 5:81-95.

5. MacKenzie, D. J., Tremaine, J. H., and StaceSmith, R. 1989. Organization and interviral homologies of the $3^{\prime}$-terminal portion of potato virus S. J. Gen. Virol. 70:1053-1063.

6. Matthews, R. E. F. 1991. Plant Virology. 3rd ed. Academic Press, San Diego, CA.

7. Rupasov, V. V., Morozov, S. Y., Kanyuka, K. V., and Zavriev, S. K. 1989. Partial nucleotide sequence of potato virus M RNA shows similarities to potexviruses in gene arrangement and the encoded amino acid sequences. J. Gen. Virol. 70:1861-1869.

8. Shukla, D. D., Lauricella, R., and Ward, C. W. 1992. Serology of potyviruses: Current problems and some solutions. Arch. Virol. Suppl. 5:57-69.

9. Titus, D. E., ed. 1991. Promega Protocols and Applications Guide. 2nd ed. Promega Corporation, Madison, WI.

10. Ward, C. W., McKern, N. M., Frenkel, M. J., and Shukla, D. D. 1992. Sequence data as the major criterion for potyvirus classification. Arch. Virol. Suppl. 5:283-297.

11. Wyatt, S. D., Druffel, K., and Berger, P. H. 1993. In vitro destabilization of plant viruses and cDNA synthesis. J. Virol. Methods 44:211-220.

12. Zavriev, S. K., Kanyuka, K. V., and Levay, K. E. 1991. The genome organization of potato virus M RNA. J. Gen. Virol. 159:373-380. 